\title{
Superficial Temporal Artery Based Pedicled Flap for the Reconstruction of Traumatic Complicated Scalp Defects
}

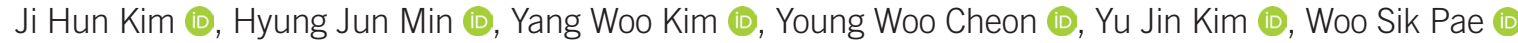 \\ Department of Plastic and Reconstructive Surgery, Gachon University Gil Medical Center, Gachon University College of Medicine, Incheon, Korea
}

\begin{abstract}
Reconstruction of traumatic defects on the scalp can be challenging. In cases of skull trauma, direct incisions close to bony defects can lead to skin and soft tissue necrosis. To minimize hair damage after surgery and to achieve both reconstructive and cosmetic effects, we propose a superficial temporal artery based pedicled flap for complicated traumatic cranial defects and lacerations. In the present case, a 21-year-old woman suffered intracranial hemorrhage with multiple skin and soft tissue injuries on her scalp after a violent attack. Neurosurgeons made a bicoronal incision for intracranial surgery and cranioplasty with a meshed plate, however $3 \times 2.5 \mathrm{~cm}$ skin and soft tissue necrosis occurred on the mid-scalp near the vertex with plate exposure. We therefore elevated a $15 \times 8 \mathrm{~cm}$-sized pedicled flap based on a unilateral superficial temporal artery and transferred it to the defect without tension. The donor site was closed in a V-Y advancement pattern. Healing was uneventful, with good hairline and hair growth patterns observed. From our experience, we suggest a superficial temporal artery pedicled flap for complicated traumatic scalp defects.
\end{abstract}

Keywords: Scalp; Temporal arteries; Perforator flap; Reconstructive surgical procedures

\section{Introduction}

The scalp covers most of the cranial skeleton with highly specialized tissues composed of dense hair follicles and inelastic, thick galea aponeurosis [1]. Tightness of the scalp makes cosmetically acceptable coverage of scalp defects challenging for plastic surgeons; therefore, reconstruction of scalp defects after oncological surgery, trauma, or burn injury is a recurring topic in reconstructive literature. Among the various methods of scalp reconstruction such as secondary healing, primary closure, skin graft, local flap, and free tissue transfer [2-4], local transposition or rotation flaps are commonly used reconstruction methods for moderate or large anterior scalp defects. Particularly for reconstruction of scalp or central forehead defects, bilateral advancement flaps are known to be the most reliable method [1,5].

In studies on structures and vascularization of the scalp and temporal areas, a variety of local flaps have been suggested for reconstruction [6-8]. In these studies, the temporoparietal fascia was found to contain the superficial temporal artery (STA), and its collateral vessels ensured the vascularization of the scalp. According to the literature, one STA is sufficient for vascularization of the ipsilateral scalp [6,8]. In addition, two STAs form an anastomotic plexus of the "full channel" type between the frontal muscular arteries and the occipital and auricular posterior arteries [8]. Hence, the use of a temporoparietal fascia flap with an STA has been introduced as a useful method in head and neck reconstructive surgery $[4,9,10]$. Moreover, this flap can pro-

Case Report

Received: July 11, 2019

Revised: October 22, 2019

Accepted: October 23, 2019

Corresponding author: Woo Sik Pae, M.D.

Department of Plastic and Reconstructive Surgery, Gachon University Gil Medical Center, Gachon University College of Medicine,

21 Namdong-daero 774beon-gil, Namdong-gu, Incheon 21565, Korea

Tel: $+82-32-460-2770$

Fax: +82-32-461-2774

E-mail: 4urdream0153@gmail.com

This is an Open Access article distributed under the terms of the Creative Commons Attribution Non-Commercial License (https://creativecommons org/licenses/by-nc/4 . O/) which permits unrestricted non-commercial use, distribution, and reproduction in any medium, provided the original work is properly cited.

C) 2020 Korean Wound Management Society 
vide healthy, durable, hair-bearing skin for patients with complicated wounds, including those from previous surgery, radiotherapy, low-grade infections, and cerebrospinal fluid leaks [10].

We herein describe a patient who received an STA-based pedicled flap surgery and present a successful soft tissue, instead of free flap, coverage method for scalp defects. This study was performed in accordance with the principles of the Declaration of Helsinki. And the informed consent was waived.

\section{Case}

A 21-year-old woman was referred to the plastic and reconstructive surgery department from the neurosurgery depart- ment. She was assaulted on her head with a blunt hammer and had multiple incision lines from the operation at the neurosurgery department. The previous intracranial surgery included dura repair and cranioplasty with a titanium mesh plate through a bicoronal incision (Fig. 1). At the time of transfer to our department, an approximately $3 \times 2.5-\mathrm{cm}$ skin and soft tissue necrosis was observed on the mid-lateral scalp area near the vertex. After debridement of the necrotic tissues, the defect was approximately $3.5 \times 3 \mathrm{~cm}$ in size, with the plate exposed (Fig. 2A). In consideration of the remaining normal tissues and characteristics of the scalp, we planned an STA-based pedicled flap for her without removing the metal plate (Fig. 2B). The entire flap was approximately $15 \times 8 \mathrm{~cm}$, including the skin paddle. The STA and its branches were identified with a
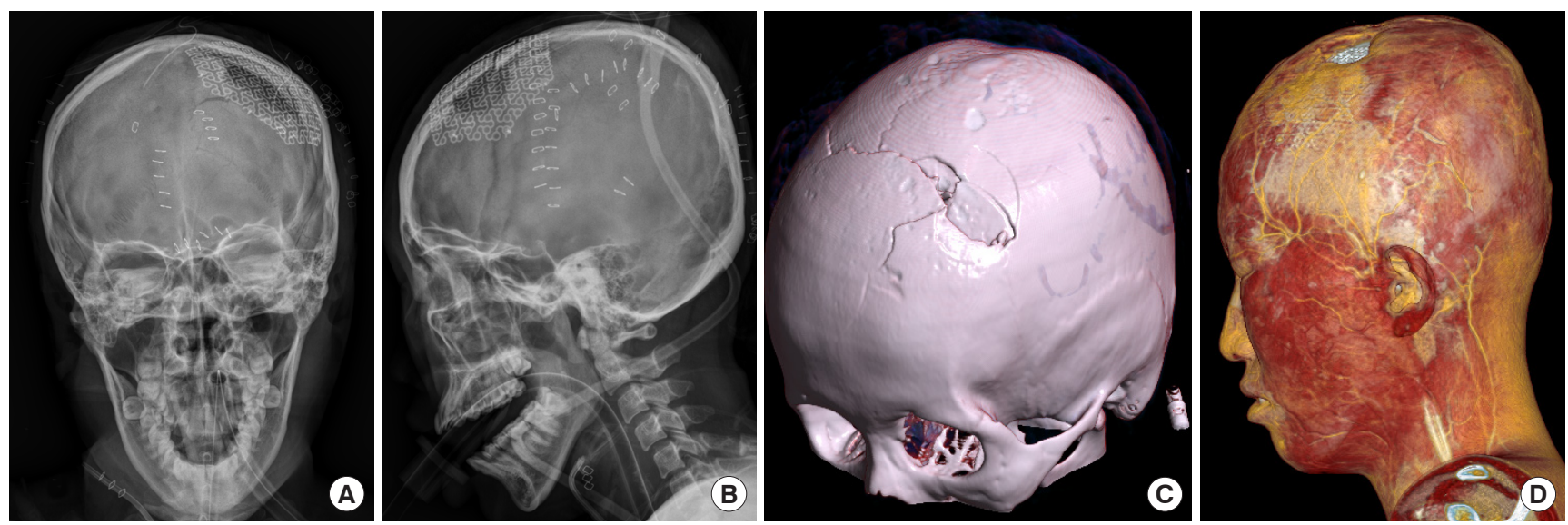

Fig. 1. Imaging study of the patient. (A) Anteroposterior view of the skull. (B) Lateral view of the skull. (C) Three-dimensional skull reconstructive image before the cranioplasty, revealing numerous multiple depressed fractures in the left frontoparietal bone. (D) Threedimensional superficial temporal artery reconstructive image.
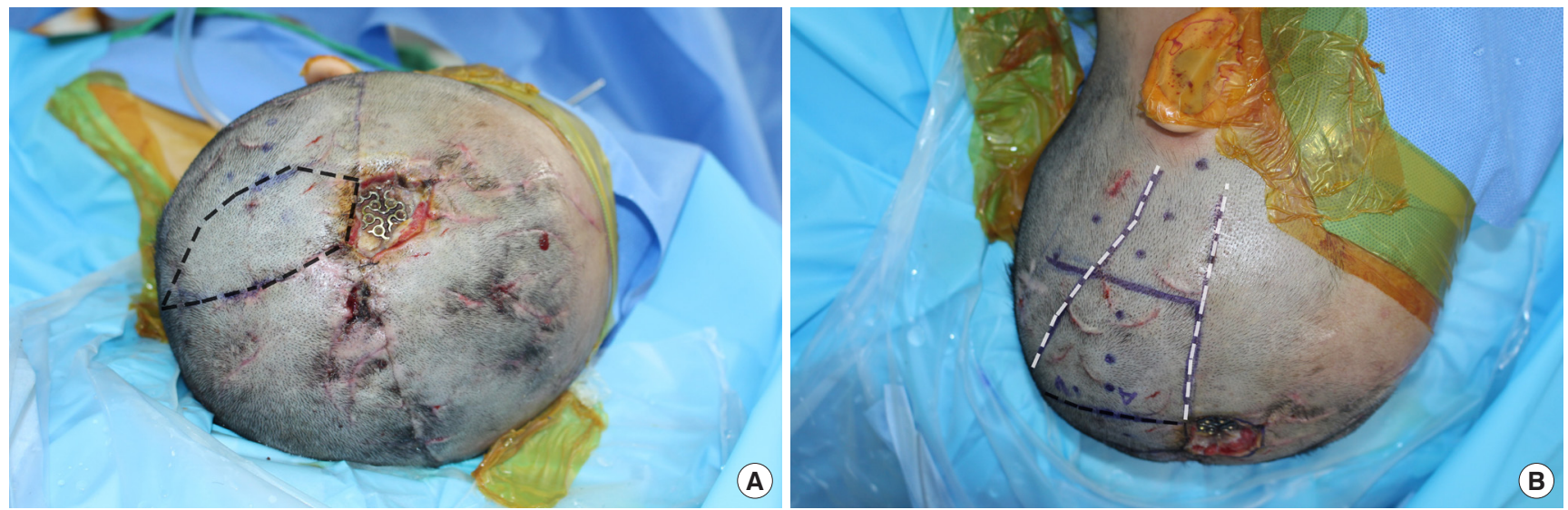

Fig. 2. Intraoperative flap design with perforator markings. (A) Intraoperative appearance of a large frontal scalp defect (3×2.5 $\mathrm{cm})$ with the metal plate exposed, after debridement of necrotic tissue. Oblong skin paddle $(8 \times 3.5 \mathrm{~cm})$ was designed to cover the defect (black dashed line). (B) We traced a superficial temporal artery on the scalp by using a handheld Doppler imaging device (marked with dots) and designed the approximate range of pedicle dissection (marked with white dashes). 

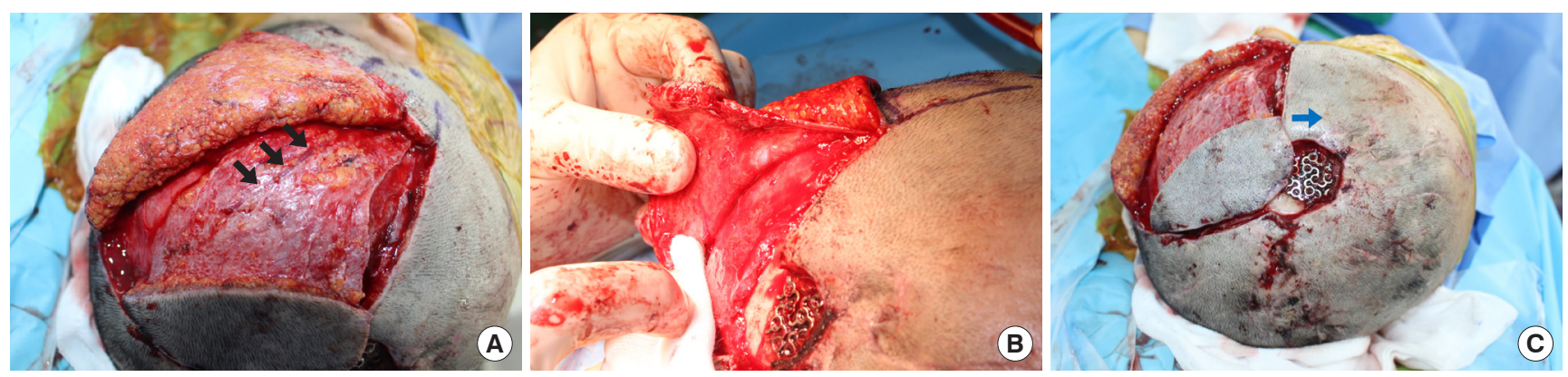

Fig. 3. Intraoperative photos of the flap elevation. (A) Subcutaneous plane dissection of the flap is performed and a superficial temporal artery is observed within the flap (black arrows). (B) Subgaleal undermining of the flap is performed and continued until the flap reaches the defect. (C) The elevated flap is moved forward (indicated by the blue arrow) to cover the defect site including the metal plate.
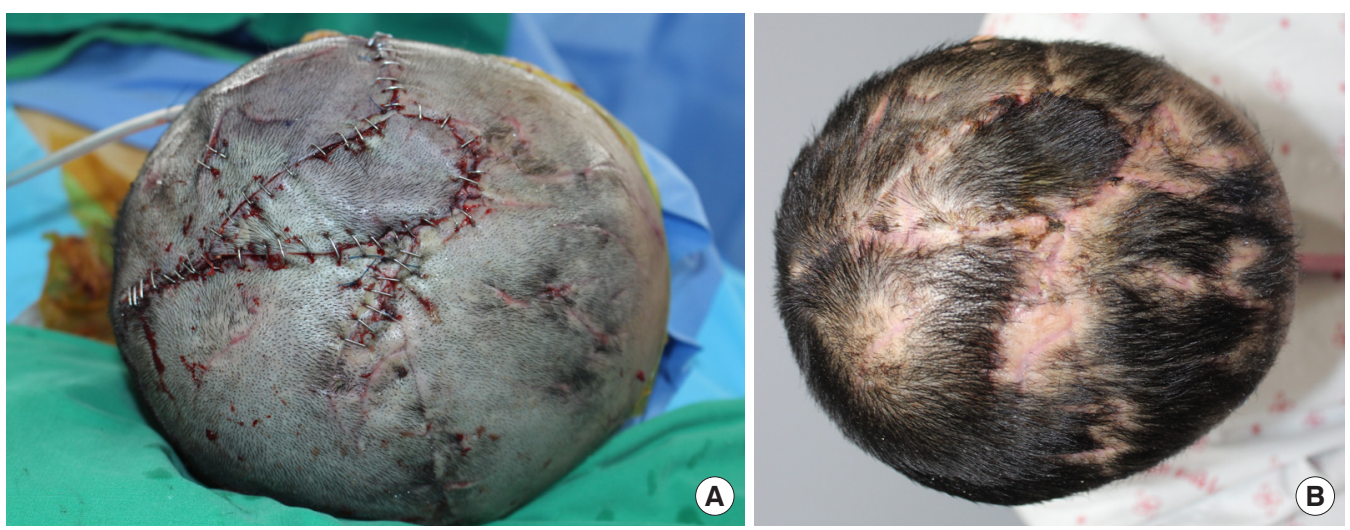

Fig. 4. Postoperative photographs of the patient. (A) An immediate postoperative photograph. The flap was $15 \times 8 \mathrm{~cm}$ and transposed from back to front to close the scalp defect without tension. The donor site and flap were closed in a V-Y pattern. (B) Healed flap at 3 weeks of follow-up.

handheld Doppler ultrasound device, and the skin flap was designed over the left temporal area of the scalp (Fig. 2). The $8 \times 3.5 \mathrm{~cm}$-sized skin paddle was designed in an oblong shape to cover the exposed plate, and an additional linear incision downward to the preauricular area was planned. After the oblong skin incision and linear incision toward the tragus were made, subcutaneous dissection was performed following the course of the artery and ending at $5 \mathrm{~cm}$ above the tragus. The STA and its branches were identified and exposed at this point, and we elevated the temporalis fascia flap from the distal aspect to the proximal aspect (Fig. 3A). The pedicled flap containing the STA with a skin paddle at the end was elevated beneath the level of temporoparietal fascia, through the subgaleal plane (Fig. 3B). Care was taken to avoid pedicle injuries throughout the procedures. The dissection was performed until the flap reached the defect without tension and the flap was moved forward to cover the defect without tension (Fig. 3C). After the rotation of the pedicled flap, the defect was covered with the skin paddle and flap insetting was done with Vicryl \#3-0 sutures and skin staples. The donor site and flap were closed with a V-Y pattern (Fig. 4A). A negative suction drain insertion and minimal compressive dressing were performed. Healing was uneventful, with good hairline and hair growth patterns observed. After 3 weeks post-operation, small patchy alopecia was observed on the previous incision lines (Fig. 4B).

\section{Discussion}

In scalp reconstruction, both anatomical features of the scalp and cosmetic elements including hair-bearing and hairline must be considered. In patients with multiple scalp traumas, the reconstruction of scalp defects is particularly difficult because of possible multiple lacerations and incision lines with bony defects. These existing lacerations and incision lines must be taken into account in scalp reconstruction.

We usually follow a reconstructive ladder when determin- 
ing surgical methods for scalp defects. Primary closure can be sufficient for small superficial defects $\left(<2 \mathrm{~cm}^{2}\right)$, but local flaps are often necessary for larger and full-thickness defects $(2 \times 25$ $\mathrm{cm})[1-3,9]$. Skin grafts or healing by secondary intention is considered for patients who refuse to undergo major reconstructive procedures. More recently, with advancements in free tissue transfer technology and physiochemical knowledge on flaps, reconstruction of skin and soft tissue defects is drawing much attention. However, transferring free tissue to reconstruct scalp defects is still challenging because of unsatisfactory aesthetic and functional results, such as poor matching with the recipient site or alopecia on the operation area.

An STA-based pedicled flap can overcome the disadvantages of free tissue transfer in scalp reconstruction and make scalp reconstruction easier for inexperienced plastic surgeons [10]. The STA flap has been described particularly to restore hair-bearing on defects of the upper lip and eyebrows [9]. Compared with free flap transfer, the STA flap limits in flap size and the distance between donor and recipient sites, and might have the complication of venous congestion [10]. However, it is considered a useful method in head and neck reconstructive surgery because of its pliability, versatility, and relatively wide pedicle rotational arc [11].

In our method, we approached the reconstruction through the previous scar and bicoronal incision line. Although the surrounding tissue of the used flap had minor damages, performing this axial flap based on one STA across a previous scar increases the risk of acute flap ischemia. Therefore, overall vascularization and STA were examined with three-dimensional angiography to confirm the STA and whether its perforators were intact before the operation. The mapping of the STA perforators was done with a handheld Doppler imaging device. In addition, to avoid hair follicle injuries, we undermined the subcutaneous fat layer after injecting it with normal saline for hydrodissection. This facilitated the dissection between the superficial temporal fascia and the hair follicle within the subcutaneous fat layer. Based on our experience, we suggest that an STA-based pedicled flap can be a useful surgical option for complicated traumatic scalp defects in certain patients.

\section{Conflict of interest}

No potential conflicts of interest relevant to this article are reported.

\section{ORCID iDs}

Ji Hun Kim

Hyung Jun Min

Yang Woo Kim

Young Woo Cheon

Yu Jin Kim

Woo Sik Pae

https://orcid.org/0000-0002-1947-2476

https://orcid.org/0000-0001-6187-9002

https://orcid.org/0000-0002-9823-9567

https://orcid.org/0000-0003-2940-292X

https://orcid.org/0000-0003-1333-4977

https://orcid.org/0000-0002-4131-7931

\section{References}

1. TerKonda RP, Sykes JM. Concepts in scalp and forehead reconstruction. Otolaryngol Clin North Am 1997;30:51939.

2. Sittitavornwong S, Morlandt AB. Reconstruction of the scalp, calvarium, and frontal sinus. Oral Maxillofac Surg Clin North Am 2013;25:105-29.

3. Frodel JL Jr, Ahlstrom K. Reconstruction of complex scalp defects: the "Banana Peel" revisited. Arch Facial Plast Surg 2004;6:54-60.

4. Leedy JE, Janis JE, Rohrich RJ. Reconstruction of acquired scalp defects: an algorithmic approach. Plast Reconstr Surg 2005; 116:54e-72e.

5. Ibrahimi OA, Jih MH, Aluma-Tenorio MS, et al. Repair of scalp defects using an H-plasty type of bilateral advancement flap. Dermatol Surg 2010;36:1993-7.

6. Abul-Hassan HS, von Drasek Ascher G, Acland RD. Surgical anatomy and blood supply of the fascial layers of the temporal region. Plast Reconstr Surg 1986;77:17-28.

7. Kleintjes WG. Forehead anatomy: arterial variations and venous link of the midline forehead flap. J Plast Reconstr Aesthet Surg 2007;60:593-606.

8. Har-Shai Y, Fukuta K, Collares MV, et al. The vascular anatomy of the galeal flap in the interparietal and midline regions. Plast Reconstr Surg 1992;89:64-9.

9. Desai SC, Sand JP, Sharon JD, et al. Scalp reconstruction: an algorithmic approach and systematic review. JAMA Facial Plast Surg 2015;17:56-66.

10. Elbanoby TM, Zidan SM, Elbatawy AM, et al. Superficial temporal artery flap for reconstruction of complex facial defects: a new algorithm. Arch Plast Surg 2018;45:118-27.

11. Kim JC, Hadlock T, Varvares MA, et al. Haira-bearing temporoparietal fascial flap reconstruction of upper lip and scalp defects. Arch Facial Plast Surg 2001;3:170-7. 